\title{
AN ELECTROMYOGRAPHICALLY CONTROLLED ELBOW LOCKING MECHANISM FOR AN UPPER LIMB PROSTHESIS
}

\author{
H.T. LAW \\ University of Edinburgh, Princess Margaret Rose Orthopaedic Hospital, Fairmilehead, Edinburgh, \\ Scotland, UK \\ and \\ J.J. HEWSON \\ Paladin Medical Products Ltd, East Kilbride, Scotland, UK
}

(Received in final form October 28, 1981)

The purpose of the elbow lock incorporated in an artificial arm is discussed and the difficulties encountered in the operation of purely mechanical devices are outlined. An electrically driven locking mechanism has been built which is controlled by the electromyogram (e.m.g.) of the surviving muscles in the upper arm. Hybrid technology is ideally suited to the construction of the associated electronic circuitry and to many similar applications now being considered in attempts to improve the performance of upper-limb prostheses.

\section{PROSTHETIC BACKGROUND}

The prosthesis (artificial arm) fitted to a patient who has undergone amputation of the arm above the elbow is illustrated in Figure 1. It consists of a carefully fitted socket placed over the stump, an elbow hinge mechanism, a forearm section and a functional "terminal device" which may be a split hook, a functional (prehensile) artificial hand or a specialised implement. The socket is held in position by a suspensory harness which passes over the shoulder, across the back and under the contralateral axilla. This harness is also used as an attachment point for the actuating cable or "motivating cord" which controls and powers the movements of the prosthesis.

The amputee is extremely limited in the variety of independent movements which he can perform using the remaining skeletal structures on the amputated side and in practice only a single cable can be usefully exercised. For this reason the active articulations are almost invariably restricted to two: those of the elbow joint and the terminal device. ${ }^{\dagger}$ The user of the prosthesis has only one of these functions available to him at any one time. Selection between the two is obtained by means of a locking mechanism incorporated with the elbow joint which allows the arm to be fixed at any one of several angles of flexion. The terminal device is fitted with a powerful spring to maintain the artificial hand (say) in a closed position. If the elbow is free to move the action of the motivating cord will, therefore, produce flexion at the elbow. If the mechanism is locked, as Figure 2 illustrates, the available work output from the motivating cord is transferred to the hand and will open it against the spring force.

\footnotetext{
†Arrangements may also be incorporated for "passive" articulations, for example to provide rotation at the wrist and at a "humeral joint", proximal to the elbow, substituting for the internal/ external rotation at the shoulder which is largely lost due to restraints imposed by the socket and suspension, but these need not concern us here.
} 


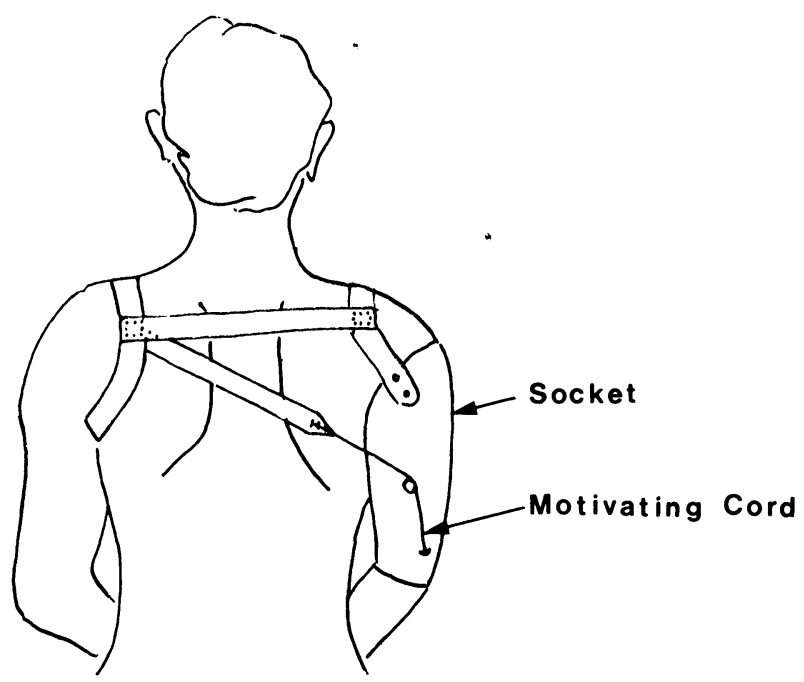

FIGURE 1 Prosthesis fitted to upper-arm amputee.

Although the locked elbow is useful in carrying heavy loads (the maximum weightlifting ability of the prosthesis positioned by the motivating cord and with the elbow free $^{1}$ is typically less than $2 \mathrm{~kg}$ ) its essential function is this transfer of the available work output, and of control, from one function to the other. It may be thought of therefore as a functional switch.

Many mechanical devices of varying ingenuity, complexity and effectiveness have been developed to provide the required switching action. The design is made difficult by the limited repertoire of independent movements of the shoulder girdle already referred to. At best only a low force, small displacement movement of the clavicle and scapula is available; this is harnessed to give control via a single pull cord. A toggle mechanism is incorporated to give a pull-to-lock/pull-to-unlock characteristic. Sadly, the majority of users of this type of prosthesis find themselves unable to master this type of control and find it necessary, therefore, to use the "good" hand to operate the lock. Obviously this detracts considerably from the functional improvement which the prosthesis brings. The smoothly progressive contraction of voluntary muscle is the result of the activity of many thousands of individual muscle cells which, when stimulated via the terminal branches of a motor nerve fibre, each undergo a contractive "twitch" lasting only a few milliseconds. This twitch is associated with a change in the permeability of part of the cell wall membrane to the ions of sodium and potassium ("depolarisation") and

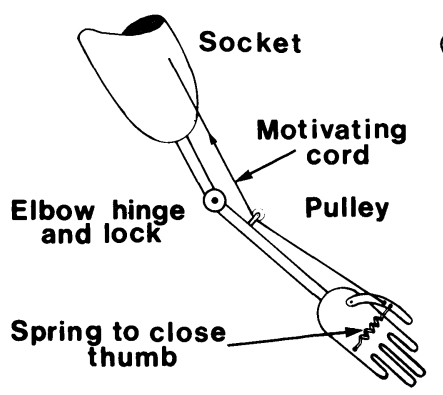

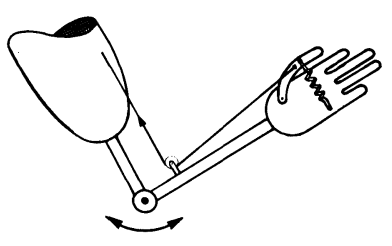

Free

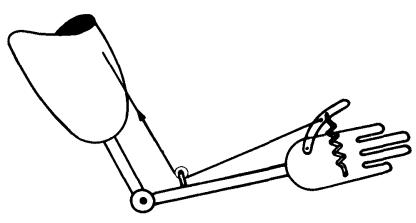

Locked

FIGURE 2 Locking mechanism component of prosthesis. 
during the event the potential difference across the cell membrane swings from its normal "polarised" value of $-90 \mathrm{mV}$ or so (negative with respect to the extracellular fluid) to about $+30 \mathrm{mV}$. The associated electric field stimulates depolarisation of adjacent parts of the membrane so that, in elongated cells such as those of muscle tissue, the depolarised region and its associated fringing electric field propagates along the length of the cell. Electrodes placed in the tissue or in contact with the overlying skin surface can be used to sense these propagating fields. Healthy muscle is electrically silent when relaxed, but during volitional effort the electrodes will detect a progressively increased electrical activity as more and more muscle cells are stimulated and, to a lesser extent, as the firing of individual "motor units" increases. The compound signal resulting from the superposition of the electrical activity of many thousands of cells within range of the electrodes constitutes the electromyogram (see Figure 3).

\section{ELECTROMYOGRAPHIC CONTROL OF PROSTHESES}

Since the muscle which powers the movement of a joint is placed proximal to that joint it may happen that, following the loss of part of a limb, the motor apparatus or muscle
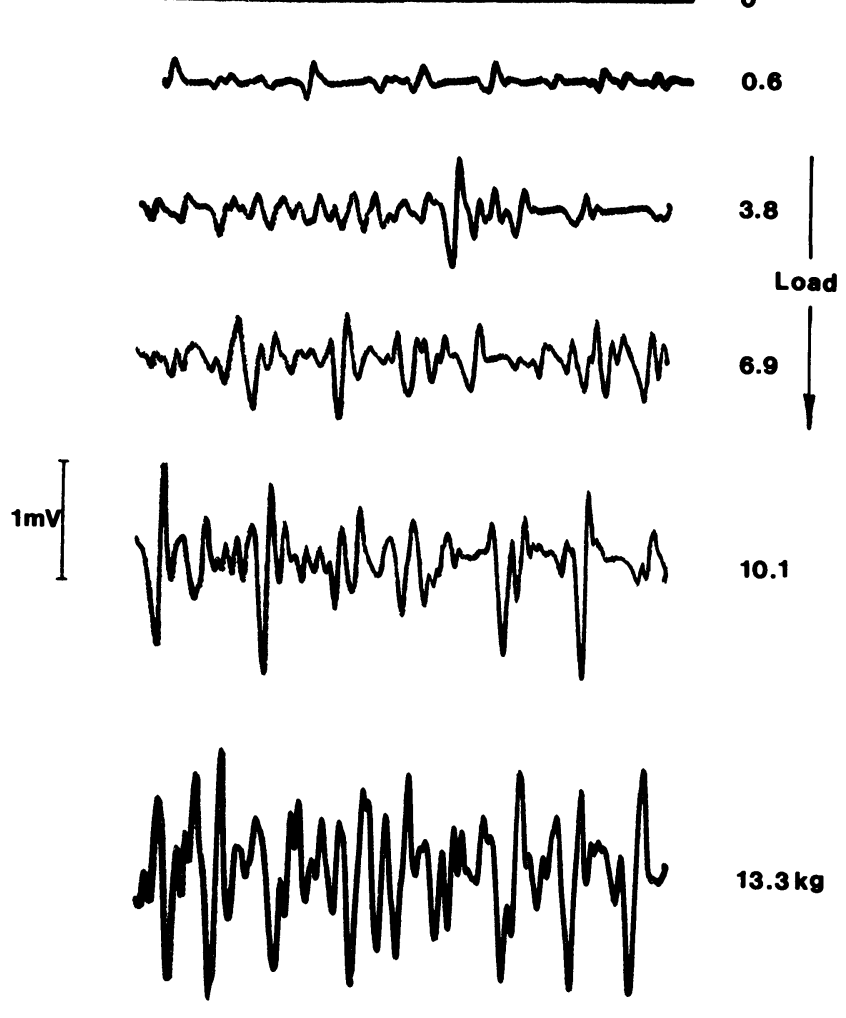

$100 \mathrm{~ms}$

FIGURE 3 Electromyogram recorded by surface electrodes over biceps muscle of normal subject with increasing isometric loading. Elbow flexed at $135^{\circ}$. 
and nerve which relates to the lost segment is retained. As soon as electronic technology provided means of detecting and amplifying the electromyogram the implications in respect of the design of a limb prosthesis became evident and as early as 1955 control of prosthetic "hands" using e.m.g. signals from forearm muscles was satisfactorily demonstrated. ${ }^{2}$

Unfortunately in following years this development failed to fulfil its earlier promise. The disappointing results are now beginning to be understood; they centre on the lack of feedback of positional information (proprioception). ${ }^{3}$ Control by means of the electromyogram provides an excellent efferent channel but no afferent nervous pathways are accessed. Physiologically this is disturbing to the user and is an important factor against the integration of the prosthesis with the body image.

\section{THE ELECTROMYOGRAPHICALLY-CONTROLLED ELBOW LOCK}

The muscles of the upper arm (biceps, triceps) in an above-elbow amputee show clearly differentiated e.m.g. signals of good amplitude. It occurred to one of us (HTL) that this could be used to control an electrically powered locking mechanism. The idea has several attractive features:

1) No movements of the prosthesis itself have to be powered so that the energy requirements are small. Good endurance from lightweight batteries can be expected.

2) The prosthesis is not made unuseable in the event of failure of the electronic package, the electrical mechanism or the power supply. Means for reversion to manual operation is easily incorporated.

3) Positional feedback (via the motivating cord) is entirely independent of the e.m.g. controlled lock. No loss of proprioceptive sense is involved.

4) Control by biceps/triceps activity is physiologically appropriate, since these muscles power the movements of the natural elbow.

The new lock is shown in Figure 4 with its mechanical precursor (a design developed at the Princess Margaret Rose Orthopaedic Hospital) shown in Figure 5 for comparison. The mechanical toggle mechanism is replaced by a permanent magnet DC electric motor (ESCAP Type 16-M11-210-0), driving the detent into engagement with the locking quadrant by means of a lead screw. The weight of the elbow assembly is increased slightly (from $400 \mathrm{~g}$ to $435 \mathrm{~g}$ ) by this change.

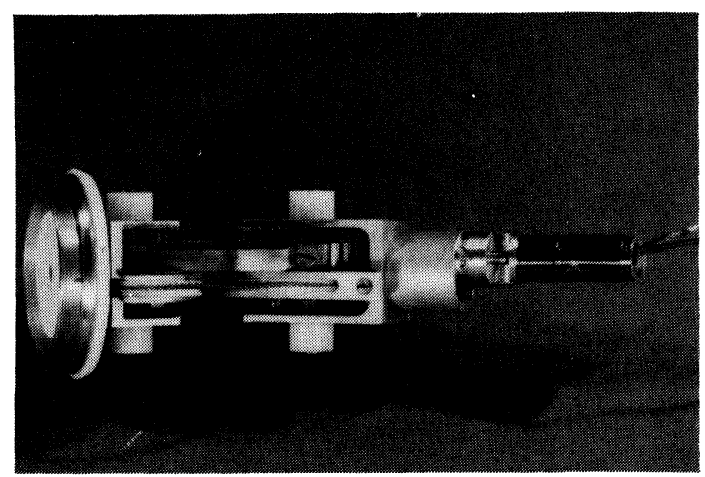

FIGURE 4 Electric elbow lock. 


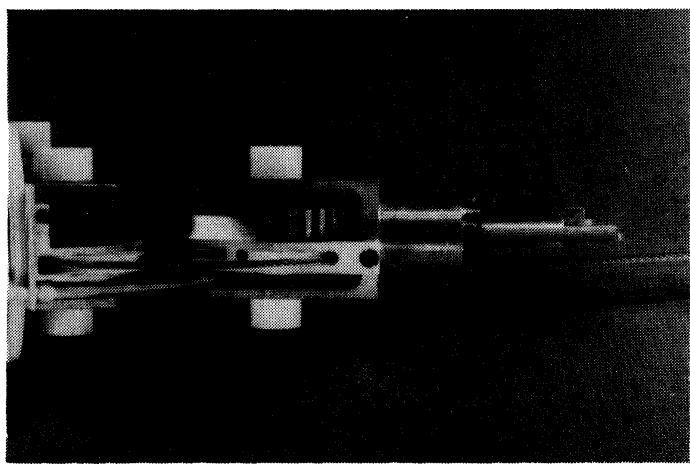

FIGURE 5 Mechanical elbow lock.

\section{THE ELECTRONIC CONTROL CIRCUIT}

The complete electronic circuit is shown in Figure 6. Its function is as follows. (Numbers relate to the sections of the circuit similarly identified in Figure 6).

1) An instrumentation amplifier used as the e.m.g. preamplifier. The gain is set by R3 (trimmed to value). For biceps e.m.g. a gain of $100(\mathrm{R} 3=1000 \mathrm{ohms})$ is suitable, giving an output of about $10 \mathrm{mV}$ r.m.s.

2) A variable gain amplifier stage (gain $1 / 3$ to 10 ).

3) The rectifier.

4) An integrator (down-going time constant $\sim 0.5 \mathrm{~s}$ ).

5) A threshold-crossing detector.

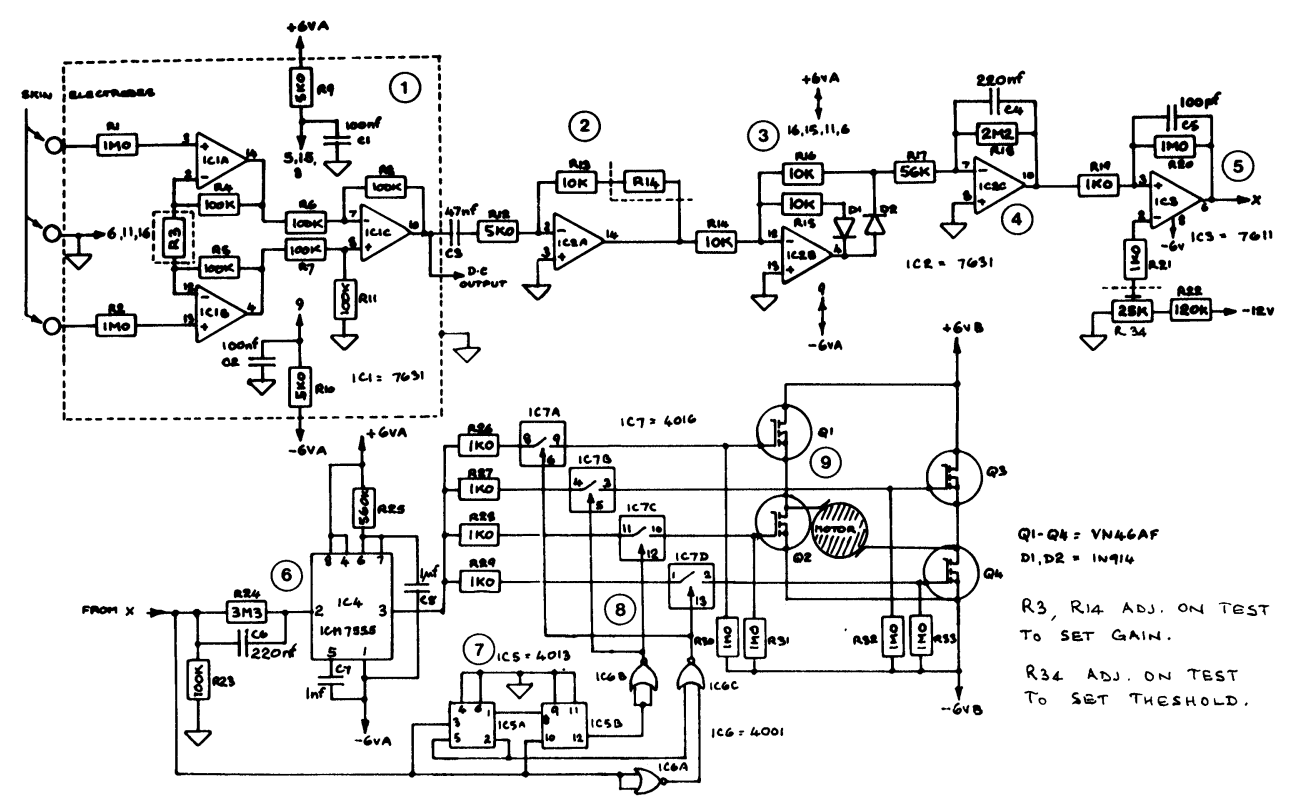

FIGURE 6 The e.m.g. amplifier and control circuit. 
6) Timing circuit.

7) Flip-flop.

8) Decoding.

9) Motor and switching transistors.

An input to the e.m.g. preamplifier of sufficient amplitude and duration will cause the output from IC3 to switch high, initiating the timing circuit and changing the state of the flip-flop. The motor will run for a preset time in the direction determined by the flip-flop. The lock is thus engaged and disengaged by successive, voluntary, brief contractions of the same (biceps) muscle. This action is similar to that of the mechanical locks and most easily learned by an established user of a prosthesis. Other control formats are possible. Using two e.m.g. preamplifiers, for instance, triceps contraction could be used to initiate elbow locking, biceps elbow unlocking. Clinical assessment is continuing to determine the control system which is physiologically the most appropriate.

\section{POWER REQUIREMENTS AND BATTERY DURATION}

The standing current in the electronic circuit is $1.6 \mathrm{~mA}$, the no load motor current is approximately $10 \mathrm{~mA}$ rising to $600 \mathrm{~mA}$ when stalled. The current waveforms during the lock and unlock events are very similar. Total duration is about $0.5 \mathrm{~s}$, total charge 58 millicoulombs. Use of the prosthesis over a 16 hour day will entail the following energy consumption:

Control circuit, $1.6 \mathrm{~mA} \mathrm{~N} 16 \mathrm{~h}$

Lock/unlock, each event 58 mA.s, 100 lock/unlock cycles each hour

$$
\begin{array}{r}
25.6 \mathrm{mAh} \\
\text { Total } \quad \sim \frac{51.6 \mathrm{mAh}}{77 \mathrm{mAh}}
\end{array}
$$

A nickel-cadmium storage battery $(2 \times 6$ volt, $80 \mathrm{mAh}$, weighing $100 \mathrm{~g}$ and volume $30 \mathrm{~cm}^{3}$ ) will suffice for a day's use. A mercury primary cell would be smaller and lighter (e.g. $100 \mathrm{mAh}, 14 \mathrm{~g}, 3.7 \mathrm{~cm}^{3}$ ).

\section{HYBRID CIRCUIT REALISATION}

The advantages which hybrid construction brings to this application are considerable. Small size and weight are obvious features (all users are aware of the weight of the prosthesis and critical of any increase) as is reliability. In many ways the environmental conditions are kindly when compared with those specified for (say) military or avionics equipment. The circuits are not required, for instance, to withstand extreme temperatures or high levels of vibration or acceleration. They will, however, be exposed to a formidable variety of pollutants (rain, salt-spray, perspiration, sand, dust ...) and extremely high standards of reliability are needed. The prosthesis is a very personal property, critically assessed. Innovative features which break down frequently will be vigorously rejected.

For hybrid construction the circuit has been divided into three parts:

(i) the e.m.g. preamplifier (section 1 , Figure 6$)$, housed in a $5 / 8$ inch square $(16 \times$ $16 \times 2.3 \mathrm{~mm}$ ) ceramic/glass/metal flatpack.

(ii) Sections $2-8$, in a 1 " square $(26 \times 26 \times 3.1 \mathrm{~mm})$ flatpack.

(iii) the power transistors, which are fitted as discrete components. 
The e.m.g. preamplifier is separated in anticipation of its use in several other applications under study at the Princess Margaret Rose Hospital and elsewhere. Also, the small, thin dimensions of the package have an important bearing on its performance as it allows good and consistent contact between the electrodes and the skin to be maintained.

\section{CONCLUSIONS}

While the "bionic arm" is still a distant, if not an unattainable, objective there is no doubt that microelectronics offers numerous opportunities for improvement in the engineering of substitutes for the human limb. The possibilities become exciting as soon as we move away from the all-mechanical prostheses which have been the conventional prescription until today.

We have described a relatively simple application of hybrid microelectronics in this novel field. We hope later to be able to report on more ambitious developments.

\section{ACKNOWLEDGEMENTS}

This development is supported under a Research Grant from the Scottish Home and Health Department.

\section{REFERENCES}

1. H.T. Law, "Engineering of upper-limb prostheses", Orthopedic Clinics of North America, 12.2, (April 1981).

2. C.K. Battye, A. Nightingale, and J. Whillis, "The use of myo-electric currents in the operation of prostheses", J. Bone and Joint Surgery, 37B, pp 506-10, (1955).

3. D.C. Simpson, "The choice of control system for the multimovement prosthesis. Extended physiological proprioception (e.p.p.)". Proceeding of the International Symposium of the Control of Upper Extremity Prostheses and Orthoses, Goteberg, Sweden, 1971. 

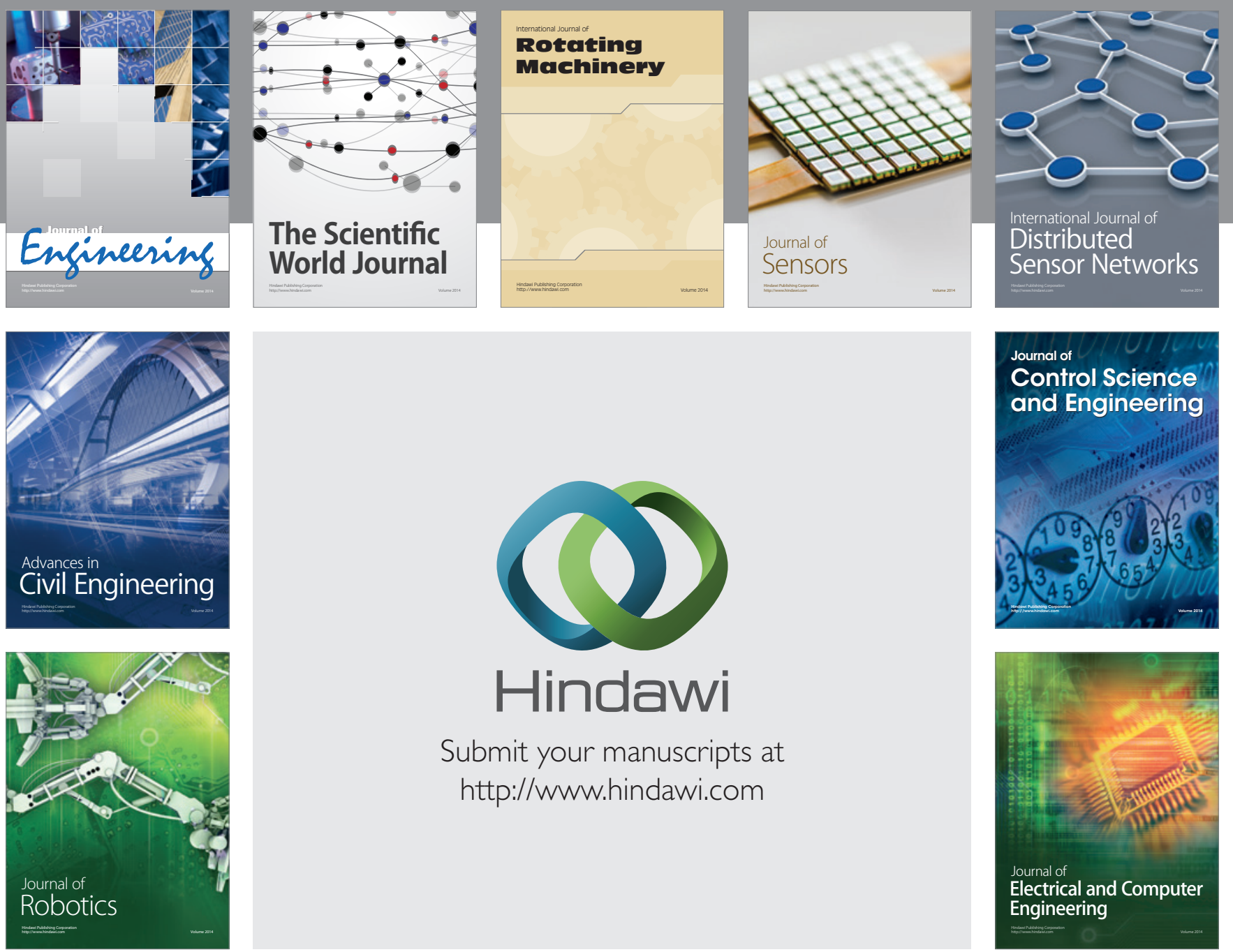

Submit your manuscripts at

http://www.hindawi.com
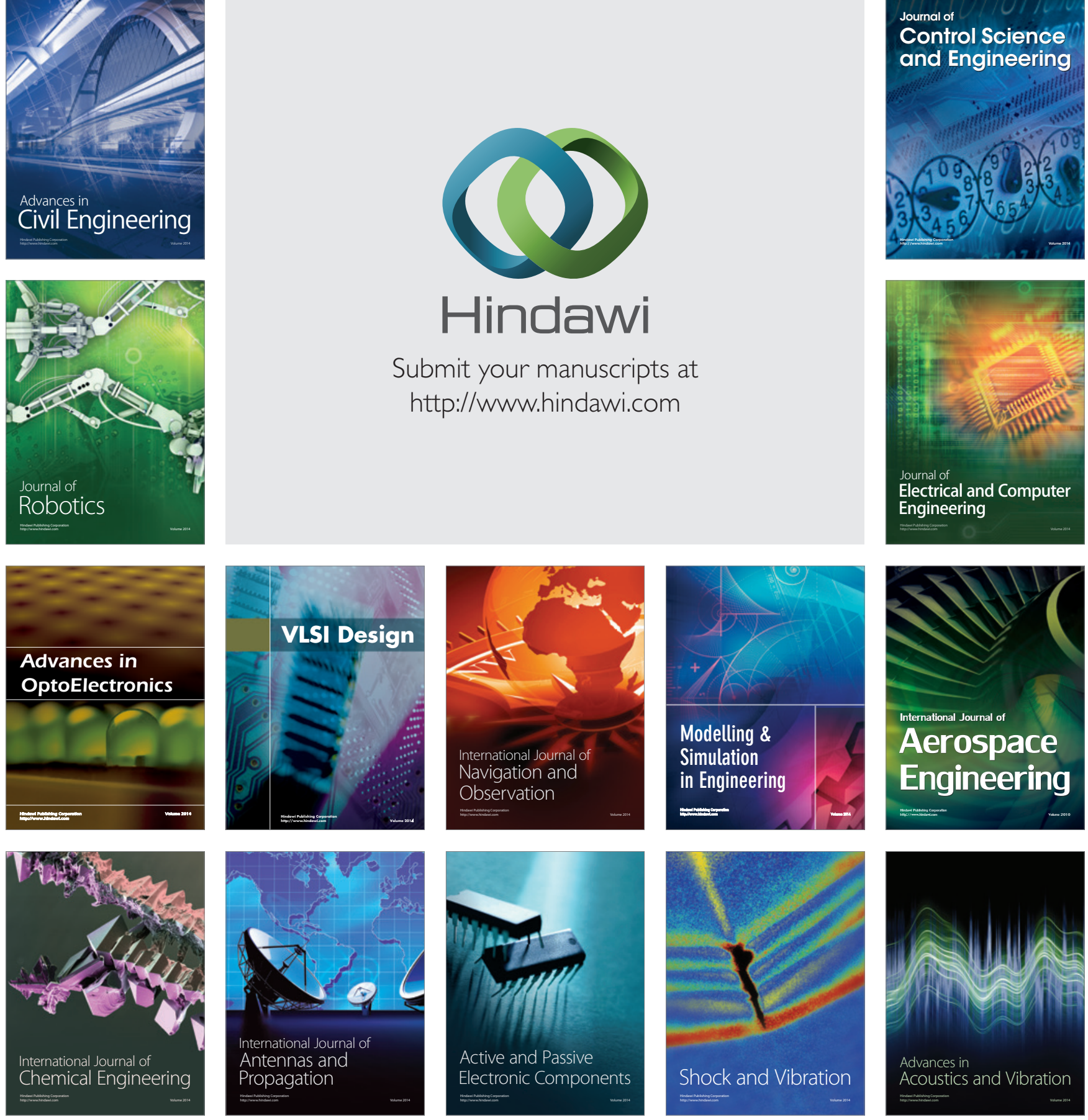